\title{
Research on Customer Profile Integration of Telecom Enterprises Based on Ontology
}

\author{
Jianlin Wu, Yan Xiong, Shuangshuang Lou and Bai Wang \\ Beijing Key Laboratory of Intelligent Communications Software and Multimedia, \\ Beijing University of Posts and Telecommunications, Beijing 100876, P.R. China \\ jlwu@bupt.edu.cn xiongyan2001@gmail.com loushuangshuang@gmail.com \\ wangbai@bupt.edu.cn
}

\begin{abstract}
In the product-centered marketing times of telecom industry, BOSS (Business Operations Supporting System) was established step by step, which resulted in the two critical difficulties in customer profile integration: semantic inconsistency and semantic conflict. In this article, ontology theory is introduced to solve these problems. Firstly, the skeleton method is adopted to build telecom customer profile ontology. Besides, a web based ontology building and query environment-Webtege is developed to give the formal definition in OWL. Then a new approach is proposed to achieve customer profile integration in telecom enterprise in both new system and legacy system scenarios from functional and data view. Therefore, this customer profile ontology could give a guideline in constituting and exchanging unified customer profile data through various systems.
\end{abstract}

Keywords: Ontology, Customer relationship management, OWL

\section{INTRODUCTION}

As the competition in global telecommunication industry becomes more and more fierce, the focus of telecommunication service is transferring from the product to the customer. However, the information systems of telecom operators are built step by step, there are two significant problems: semantic inconsistency and semantic conflict, which decreases the satisfaction of customer services.

The problem of semantic inconsistency has two dimensions: first, because the different systems are built in different times, the systems can not reach an agreement on the information model; second, in the information models, the attributes of table with the same meaning have different codes, resulting that even though various systems have the same logical models, data can not be understood and exchange by different systems. On the other hand, the problem of semantic conflict also has two dimensions: first, the customer information is short of verification; second, the customer information in different systems is updated in different time, so the profiles of one customer in different systems are quite various.

It is critical to handle the above two problems for the integration of customer information of telecommunication enterprises. Ontology can effectively describe the various concepts and the relationships between them on the semantic and knowledge 
level, leading to the share of the concept relationships among various systems. This paper tries to utilize the ontology model methodology to solve the mentioned semantic problems. First, we establish the customer profile ontology of telecommunication operators to achieve the agreement on customer profile models. Then we use the established customer profile ontology to handle the semantic heterogeneous and conflicting problems.

\section{RELATED WORK}

Information integration could be viewed as there levels: physical, logic and semantic. In physical and logic information integration level, technologies such as COM, CORBA and Agent, have also been developed to solve the technical problem of information integration [1].

In semantic level, many efforts are made. Wang [2] gives the structure, consistency checking and development process of ontology-based metadata model whose research is supported by National Nature Science Foundation of China: Customer Ontology in CRM Study. In his dissertation, Wang tries to establish simple customer ontology to be applied in all industry. In this research group, Lu [3] proposes customer features extraction based on customer ontology; Wang [4] makes efforts on the mechanism of information retrieval based on customer ontology. The above mentioned research is devoted in the common customer information ontology or integration metadata, but there is also customer ontology research in specific domain. Zhu [5] makes research on customer information sharing in the supply chain of automobile. Yan [6] establishes contract ontology in OWL to express semantic relations. Huhns [7] build personal ontology to filtrate information in the web. However, when we refer to telecom industry the ontology concept is rarely used to settle the specific problem of information integration. Here is the meaning of this paper-introducing ontology to telecom industry.

\section{BUILDING CUSTOMER ONTOLOGY}

\subsection{Methodology of Building Customer Ontology}

Ontology was original a philosophy concept which is the description of concepts and relationship between concepts. The application of ontology experiences the development from philosophy to artificial intelligent area and is now widely used in information and other areas [8]. Studer [9] defined Ontology as explicit formal specification of shared concept model in 1998. The language describing ontology could be divided as based on framework and logic. The language OWL [10] used in this article is one description language based on logic. Skeleton method is also called Ushold and King Method [11], which is derived from Enterprise Ontology building 
experiences by Edinburgh University in England. There are four steps to develop the ontology using skeleton methodology: 1. Define ontology objective and scope; 2 . Building ontology, including concept extracts and define, codify and integration exists ontology; 3. Evaluation, evaluate the build ontology in technical aspect; 4. Documentation, suggestion about strategy and effection of the building ontology.

\subsection{Objective and Scope}

In this paper, we circumscribe the scope of ontology building on telecom operator customer. As described in SID (Shared Information Data) [12], the overall information in telecom enterprise could be divided into eight domains. Here we restrict the discussion of data and information in customer domain. According to eTOM (enhanced Telecom Operation Map) [13], telecom customer information could be viewed as three themes: Customer, Subscriber and Account. The definition of Customer and subscriber could be found in eTOM documents. We give definition of Customer Account. See table 1 for the definition and characteristic of those three important concepts which are also our main objectives in ontology building.

Table 1. Customer, Subscriber and Account' Definition and Characteristics

\begin{tabular}{|l|l|l|}
\hline Concept & \multicolumn{1}{|c|}{ Definition } & \multicolumn{1}{|c|}{ Characteristic } \\
\hline Customer & $\begin{array}{l}\text { The Customer buys products and services from } \\
\text { the Enterprise or receivers free offers or } \\
\text { services. A customer may be a person or a } \\
\text { business.[13] }\end{array}$ & $\begin{array}{l}\text { Social related data of a person or a } \\
\text { company }\end{array}$ \\
\hline Subscriber & $\begin{array}{l}\text { The Subscriber is responsible for concluding } \\
\text { contracts for the services subscribed to and for } \\
\text { paying for these services. [13] }\end{array}$ & $\begin{array}{l}\text { Usage related data generated when a } \\
\text { customer subscribe or use a product or } \\
\text { service }\end{array}$ \\
\hline Account & $\begin{array}{l}\text { The Account is owing to one or several } \\
\text { product usage. }\end{array}$ & $\begin{array}{l}\text { Finance related data generated when a } \\
\text { customer deposits cash in telecom operator } \\
\text { in order to pay for usage of products }\end{array}$ \\
\hline
\end{tabular}

\subsection{Customer Ontology}

As discussed before, there are four criteria to follow when we are building the ontology: conceptualization, explicit, formal and share. We adopt these criteria for our telecom customer ontology. Here, we define two kinds of entities in the customer ontology: Substantial Entity and Information Entity.

Definition 1:

Substantial Entity: Substantial Entity is a kind of entity representing a substantial concept which could refer to a corresponding role in the real physical world.

Definition 2:

Information Entity: Information Entity is a kind of entity which represents information concept or a set of data which could not refer to a corresponding role in the real physical world but are generated according to the substantial entity activities. 


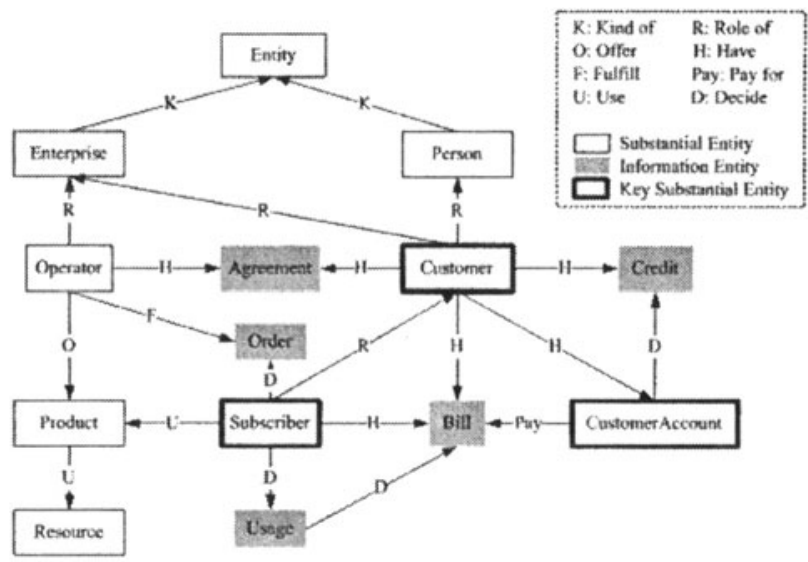

Figure 1. Concept-Relationship Description of Telecom Customer Ontology

For the authors' experiences in telecom industry and the study into information model from many telecom operators, we could abstract the concepts and relationship as Figure 1. We have nine substantial entities, five information entities and eight kinds of relationships in telecom customer ontology. Key substantial entity represents the three theme model which is the centre of the whole model.

Each relationship connects two entities: from domain to range. Relationship $K$ : Kind of implies the inherited characteristic from some upper class entity like Subclass of. $R$ : Role of also implies Subclass of relationship, but different from Kind of that it means an entity performed especially in a particular operation or process. $O$ : offer means provide in performance. $H$ : Have means hold or maintain as a related information entity. Pay: Pay for means make due return to for services rendered. $U$ : Use means the act or practice of employing some services. D: Decide means the activities of domain substantial entity would determine the data in information entity. $F:$ Fulfill means to meet the requirements of a business order.

Besides entity and relationship, the attribute is also an important part of ontology model. Here we list the $2^{\text {nd }}$ level entity and attributes of the entities above. These may not include all the attributes since the limited pages of this article.

\subsection{OWL Description of Customer Ontology}

As the telecom customer ontology we put forward, we choose OWL as a formal description language in this paper. We use the ontology building and query environment based on web technology-Webtege which is developed by our research team recently. It provides the user graphic webpage to build a new ontology model, convert it into OWL files and query certain relationship or entity in the ontology. Figure 2 are pictures of customer ontology and OWL files built in Webtege. 
Table 2. Concept-Attributes Description of Telecom Customer Ontology

\begin{tabular}{|c|c|c|}
\hline Entity & 2nd level Entity & Attributes \\
\hline \multirow{2}{*}{ Customer } & Personal customer profile & $\begin{array}{l}\text { Name, Gender, Age, Vocation, Contact, Security ID, Affiliation, } \\
\text { Customer level }\end{array}$ \\
\hline & Enterprise customer profile & $\begin{array}{l}\text { Enterprise customer number, Address, Customer manager, } \\
\text { Status, Level }\end{array}$ \\
\hline \multirow{2}{*}{ Subscriber } & $\begin{array}{l}\text { Basic services related } \\
\text { information }\end{array}$ & $\begin{array}{l}\text { Telephone number, SIM, Package fee used, Terminal unit type, } \\
\text { Personal services, Fulfill channel, Status }\end{array}$ \\
\hline & $\begin{array}{l}\text { Value-added services } \\
\text { related information }\end{array}$ & Service type, Preferential plan \\
\hline \multicolumn{2}{|c|}{ Customer Account } & $\begin{array}{l}\text { Account ID, Name, Type, Status, Valid period, Balance, Contact } \\
\text { person, Telephone, Open time, Calling up of balance }\end{array}$ \\
\hline \multicolumn{2}{|r|}{ Agreement } & $\begin{array}{l}\text { Customer Service Level Agreement, Type, ID, Responder, } \\
\text { Responsibility person, Customer responsibility person, Start } \\
\text { time, Expire time }\end{array}$ \\
\hline \multicolumn{2}{|r|}{ Order } & Order ID, Order association \\
\hline \multicolumn{2}{|r|}{ Credit } & Customer credit information, Customer credit history \\
\hline \multicolumn{2}{|r|}{ Bill } & Bill format, Bill type, Bill requirement, Bill entry, Bill receipt \\
\hline \multicolumn{2}{|r|}{ Usage } & Local call record, Long term call record, Roam call record \\
\hline
\end{tabular}
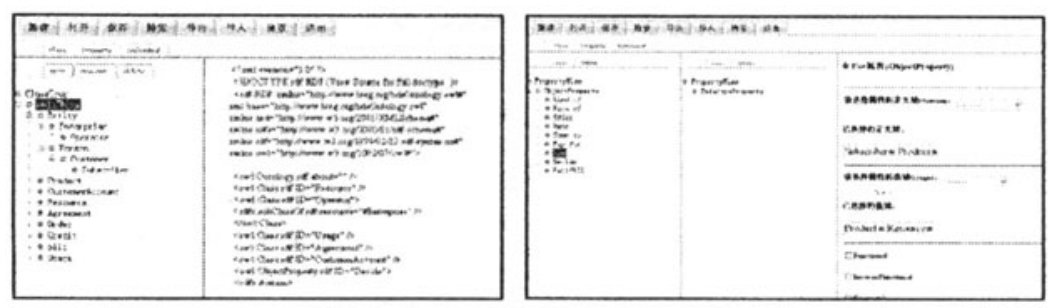

Figure 2. Customer Ontology and OWL Description in Webtege

\section{CUSTOMER PROFILE INTEGRATION BASED ON ONTOLOGY}

The purpose of customer ontology is to reach the integration of customer profile in telecom enterprise. The customer ontology is a kind of domain ontology and thus reach an agreement on customer profile understand throughout the different enterprise in telecom industry. Here, we propose a new method to achieve customer profile integration based on the customer ontology. We divide this problem into two scenarios: the integration in new systems and the integration in legacy systems.

\subsection{Customer Profile Integration in New Systems}

Once the customer ontology is approbated among telecom domain, the customer profile in new systems would be built under the direction of customer ontology. We present the integration process in both functional view and data view. 


\subsubsection{Functional View}

In functional view we describe the mainly steps for integration. It may not contain the detail tech but the approach or in another words technique roadmap to achieve the integration purpose. As described in Figure 3, there are three steps to fulfill the customer profile integration in new systems: Generating, Directing and Restricting. In generating step we convert the customer ontology to customer profile standard metadata, i.e. E-R model for customer profile in logic level. The detail principles of generating would be presented in the data view below. Then in directing step we take E-R model into standard table, i.e. data structure in physical level. Finally, restricting step is used to make sure the data in table would follow the restrictions of E-R model and further the ontology constrains. For example, the restriction would be the data type of each row of table; constrains would be the union of, disjoint with and etc. restrictions between entities of customer ontology. Therefore, the problem of semantic conflicts would be settled.

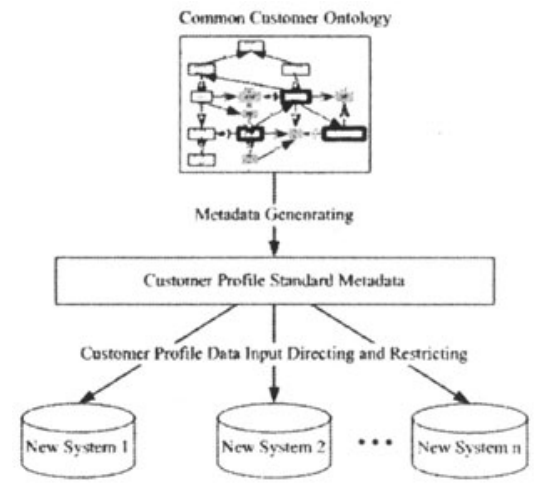

Figure 3. Customer Profile Integration in New systems-Functional View

\subsubsection{Data View}

The customer ontology built in section 3.3 of this article is a prototype ontology base. While applying it in the real circumstance, we need more detailed information in table or attributes level. Here, in data view we take four entities in ontology for example to show how essential tables are determined according to ontology. We focus on Customer, Customer Account, Credit and Bill ontology. In generating step we makes mapping from ontology to E-R model. Here we define two rules for the mapping:

Generating Rule 1:

Entity generating: One entity in ontology could correspond to at least one entity in E-R model which should express complete information of that entity.

Generating Rule 2:

Relation generating: One relationship in ontology could correspond to one or more relation in E-R model. 
As we describe in Figure 4, four entities in ontology correspond six entities in E$\mathbf{R}$ model. The relations are draw as diamonds which is the same as relationship described in ontology. In directing step we detail the E-R model into data table format in physical data level. The rules could be found in database principles of book [14]. In the example here in E-R model three entities--Customer, Personal Customer, Company Customer, could refer to eight tables. The attributes in the table are expanding to the attributes of entities in ontology. In restricting step we take additional vocabulary of ontology such as disjointWith, intersectionOf and etc. for the purpose of expressing more semantic relations and restrictions such as disjoint, intersection, union, complement, enumeration, property restrictions, and cardinality into constrains for table. For example, if we define the maxCardinality of relationship "have" from Customer to Customer Account as 10, and then the relationship table should reflect this cardinatlity. Through these steps, guidance could be given from ontology to table building. Therefore, the customer profile table in new systems could be build according to a unified standard. So we could achieve the integration in this new system scenario.

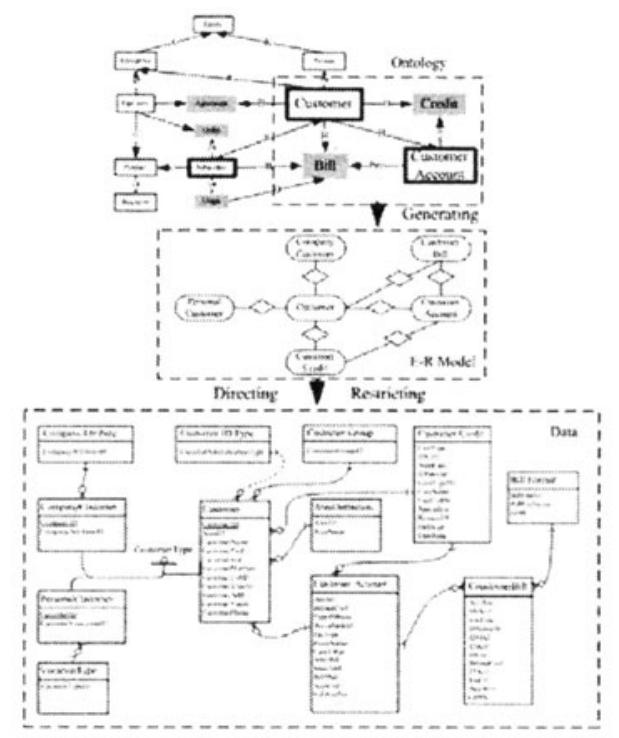

Figure 4. Customer Profile Integration in New systems - Data View

\subsection{Customer Profile Integration in Legacy Systems}

Besides the new system scenario mentioned above, there exists difficulties in customer profile integration among different legacy systems. Now in telecom 
business and operating systems, the established customer profile does not conform to certain unified standard. We take advantage of the above customer ontology to solve these problems. We also present the integration process in both functional view and data view.

\subsubsection{Functional View}

As described in Figure 5, there are four steps to fulfill the customer profile integration in legacy systems: Extracting, Generating, Mapping and Restricting. The Generating and Restricting steps would be the same as described in 4.1.1. Here we describe the Extracting and Mapping steps.

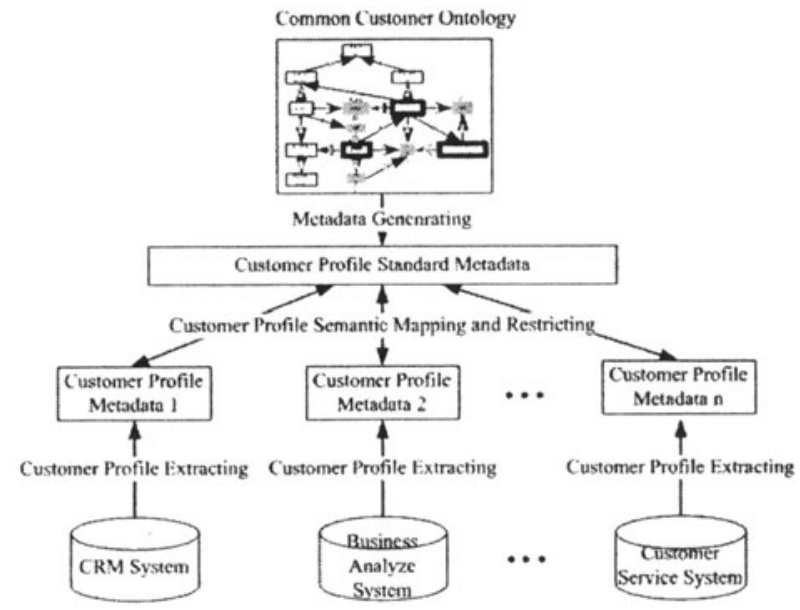

Figure 5. Customer Profile Integration in Legacy Systems-Functional View

In Extracting step we take customer related metadata from legacy systems such as CRM, Business Analyze System, Customer Service System and etc. We can extract metadata according to customer theme or domain from the database's logic model. Or we can use extract tools such as Powermart [15], a data extracting tool from Informatica Inc., to get the metadata. Then in mapping step, we compare the metadata extracted from legacy system to the standard metadata generated from ontology. The detailed mapping explanation refers to 4.1.2. After the comparison of metadata, the legacy metadata might be converted into part of standard metadata, or expand the standard one on the base of which customer data exchange between different systems would be realized and thus solve the problem of semantic inconsistency.

\subsubsection{Data View}

As in Figure 6 the generating and restricting steps of data view of customer profile integration in legacy systems are the same as described in 4.1.2. The extracting step is the reverse operation of directing step in 4.1.2. Its rules could be found in database 
principles-abstract physical level data to logic level data. The mapping step might relate to some semantic aspects since the metadata might have the same meaning but use different name. For example, the attributes record Customer universal identification in Customer table of standard metadata might be marked as "CusID", but in legacy system the corresponding attributes might be marked like " $\mathrm{C}$ id".

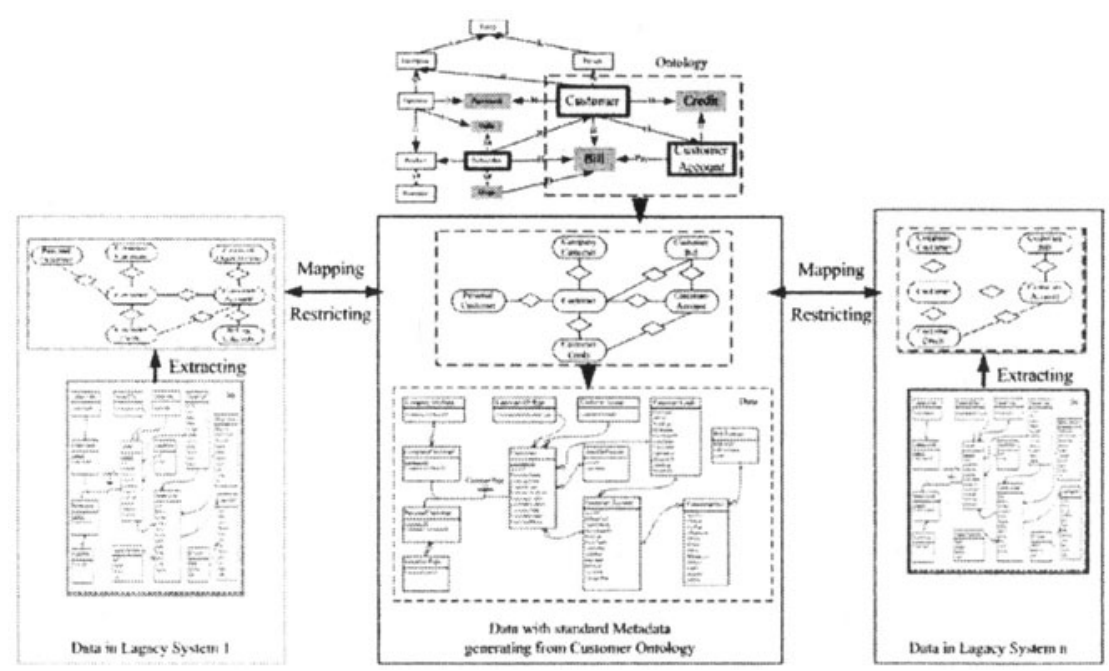

Figure 6 Customer Profile Integration in Legacy Systems-Data View

\section{CONCLUSIONS}

In conclusion, the building of telecom ontology has significant bearing in solving the semantic inconsistency and semantic conflict problem and thus in realizing the customer profile integration. The customer ontology and integration approach proposed in this paper could give a guideline in constituting customer profile in new systems and achieving the integration over various legacy systems. As a result, according to customer service-centered goal of telecom enterprise, customer profile integration could make the execution of customer relationship management strategy more effectively and thoroughly and the integrated customer information could be (re)used and analyzed for diverse marketing purposes in the coming $3 \mathrm{G}$ times of China.

In the future, first we are going to reify, evaluate and improve our telecom customer ontology by using them in the real situation, inviting other telecom industry peers to evaluate our ontology. Second we are going to propose more detailed feasible technique to realize the integration approach and develop a demo to verify. 


\section{REFERENCES}

1. R.E. Filman, D.J. Korsmeyer, and D.D. Lee, CORBA Extension for Intelligent Software Environments, Advances in Engineering Software. Volume 31, Number 8, pp.727-732, (2000).

2. H. Wang, Research on the Construction of Metadata Models Based on Ontology. Ph.D Thesis, Shanghai Jiao Tong University (2004).

3. X. Lu, F. Jiang, and L. Hou, Customer Features Extraction Based on Customer Ontology, Computer Engineering. Volume 31, Number 5, pp.31-33, (2005).

4. F. Wang, Research on the Mechanism of Information Retrieval Based on Customer Ontology. Master Thesis, Shanghai Jiao Tong University (2005).

5. J. Zhu, J. Wu, and F. Jiang, Study on Customer Information Sharing in the Supply Chain of Automobile, Information Science. Volume 23, Number 7, pp.1094-1097, (2005).

6. Y. Yan, J. Zhang, and M. Yan, Ontology Modeling for Contract Using OWL to Express Semantic Relations, in Proceedings of the 10th IEEE International Enterprise Distributed Object Computing Conference (EDOC'06), eds. B. Werner (IEEE Computer Society: New York, NY, 2006), pp.409-412.

7. M.N. Huhns and L.M. Stephens, Personal Ontologies, Internet Computing. Volume 3, Number 5, pp.85-87, (1999).

8. Z. Deng, S. Tang, and M. Zhang, Overview of Ontology, Acta Scientiarum Naturalium Universitatis Pekinensis. Volume 38, Number 5, pp.730-738, (2002).

9. R. Studer, V.R. Benjamins, and D. Fensel, Knowledge Engineering, Principles and Methods, Data and Knowledge Engineering. Volume 25, Number 122, pp.161-197, (1998).

10. M.K. Smith, C. Welty, and D.L. McGuinness, OWL Web Ontology Language Guide, W3C (2004). http://www.w3.org/TR/2004/REC-owl-guide-20040210/ (Accessed May 20, 2007).

11. M. Uschold, M. King, S. Moralee, and Y. Zorgios, The Enterprise Ontology, The Knowledge Engineering Review. Volume 13, Number 1, pp.31-89, (1998).

12. TM Forum, Shared Information/Data (SID) Model Addendum 2 Customer Business Entity Definitions Release 6.0 (TeleManagement Forum: Morristown, NJ, 2005).

13. TM Forum, Enhanced Telecom Operations Map (eTOM), The Business Process Framework Release 6.0 (TeleManagement Forum, Morristown, NJ, 2005).

14. A. Silberschatz, H.F. Korth, and S. Sudarshan, Database System Concepts Fourth Edition (McGraw-Hill: Columbus, $\mathrm{OH}, 2001$ ).

15. Anonymous, Data Integration Software - Informatica Products, Informatica Inc., (2007). http://www.informatica.com/products/default.htm (Accessed May 20, 2007). 